\title{
Research on the Coordination of Green Finance and Green Economy with the Goal of Sustainable Development
}

\author{
Bing Yang \\ Minsheng Investment Management Company, Beijing 100005, China \\ Email: 344097812@qq.com
}

\begin{abstract}
In order to give full play to the integration between green finance and green economy, the selection of appropriate evaluation indicators is worth further exploration. Therefore, this paper is conducted with exploration on the combination of comprehensive index system and comprehensive method, which give evaluations on the coupling coordination degree between green finance and green economy by combining the coupling coordination degree model with ESG, so as to achieve the goal of sustainable development.
\end{abstract}

Keywords: green finance, green economy, coupling coordination, carbon asset management, ESG

\section{Introduction}

Environmental problems such as ecological imbalance, resource exhaustion and environmental pollution are closely related to social development and have become global economic and political problems. Early researches on green finance and green economy were given major focuses on the theoretical analysis of concept and system structure, and then gradually the concept of sustainable development was introduced into financial practice. Some countries have begun to conduct overall research on the green efficiency in the business development of financial service institutions, and financial service institutions were guided to implement the principles of green finance and better fulfill their social responsibilities, so as to play the role of green finance in economic ecological development and improve the coordination between green finance and green economy.

There are good practices in the world for the coordination of green finance and green economy. From the regulatory perspective, in order to mainstream the concept of global sustainable development, some countries have incorporated climate change and green finance into their financial regulatory policy framework. From the perspective of investment, the realization of green finance is also the demand of medium - and long-term capital investment to avoid risks. From the perspective of volatility, the green bond index and carbon market can effectively identify the volatility of green bond market and carbon asset pricing. From the perspective of fund raising, the ways of green fund raising show a trend of diversification. From the perspective of green economy, the green development and transformation of industry can be further promoted through the joint action of environmental benefits, social benefits and economic benefits as well as policy guidance. In the future, we are also exploring the application of ESG in the daily operation and management of enterprises from the perspective of environment, society and governance, so as to promote the implementation of green finance and the transformation of green economy.

\section{Development status of green finance and green economy}

Problems such as environmental pollution, climate change and biodiversity loss were once regarded internationally as the inevitable consequences of economic growth. Since the United Nations Conference on Environment and Development in 1992, enterprises have paid more attention to the balanced development between economy and environment, society and governance in the process of operation and management to achieve the sustainable development goals. Therefore, the goals in environmental and climate protection have become an important topic in the economic development based policies of various countries, and green growth has become another perspective of green finance and green economic development. The Organization for Economic Co-operation and Development (OECD) points out that green growth is capable of promoting economic growth and development and ensuring that natural resources can continue to provide the environment on which human survival depends. The World Bank has also defined green growth, which can achieve both traditional economic growth and efficient use of natural resources to minimize the impact on the ecological environment. At present, the green and low-carbon transformation is changing the global competitive landscape, prompting policymakers to pay more attention to the coordinated development of green finance and green economy.

Data show that there is an inverted U-shaped relationship between the financial development and economic growth. In order to achieve efficiency optimization, the establishment of scientific green financial evaluation system is an important way 
to promote the development of green economy. However, due to the lack of a clear standard of quantitative and statistical data, there are many problems on the study on evaluation on green finance, part of studies on the cases with the economic and environmental benefits split most countries, regions and business entities in the description and analysis of financial and economic coordinated development in the past and present, which ignores the interaction and influence on the space. There are few studies on the distribution and dynamic evolution of coordination degree considering the factors such as spatial spillover.

An important support for green growth is the development of environmentally beneficial technologies and financial products. Diversified paths of green support indicate that climate change mitigation efforts are largely dependent on the strength of financial support. For example, green credit can be provided to support the improvement and deployment of renewable energy technologies. Applying risk monitoring and rating techniques to the sustainable management of ecological resources. The issuance of carbon bonds to support the carbon cycle of enterprises plays a key role in reducing the environmental impact of their production processes. By providing a variety of low-carbon financial products, it can promote the development of low-carbon and circular economy and achieve the goal of carbon neutrality. Natural carbon sinks are important for achieving net zero emissions and can promote sustainable afforestation, reforestation, agricultural soil management and peatland restoration. Therefore, in order to succeed in the green economy of the future, it is necessary to build the green financial evaluation system earlier and more actively, and to build the green production capacity.

\section{Interaction between green finance and green economy}

Financial development is closely related to economic growth, and green finance and green economy show close pertinence. Therefore, we need to pay attention to the coordination between economic growth and environmental protection. Generally speaking, financial markets and service institutions that play an increasingly important role in sustainable development should pay full attention to this relationship. A good development environment will also help financial institutions better and effectively play their roles in society and economy, so green finance is an important measure to achieve low-carbon green growth. There are two reasons for this. On the one hand, it links financial development, economic growth and environmental improvement. On the other hand, as a new financial model combining environmental protection and economic benefits, it can effectively solve the problem of market failure, and strengthen the prevention before the occurrence of risk events and the immediate supervision and management when the event occurs.

The interaction between green finance and green economy is conducive to the sustainable development of social economy. For China, there is still a gap in the coordinated development of green finance and green economy among various provinces. Therefore, in order to achieve balanced development, it is necessary to strengthen the construction of diversified green financial systems, such as the development of green bonds, carbon asset management, ESG financial products, energy conservation and environmental protection venture capital funds, and avoid the transfer of polluting industries to areas with weak environmental and ecological carrying capacity

In practice, we need to realize the coupling and coordination between green finance and green economy to realize the comprehensive coordination between regions, promote green finance and green economy, and realize the sustainable development of the whole society of the country. It is important the changes in one area can not be the only focus, rather the coordinated development of the two should be the goal in policy.

\section{Indicator system for green finance and green economy}

In August 2016, seven ministries and commissions of the State issued the Guiding Opinions on the Construction of green Financial System, making it clear that we should accelerate the transformation of green economy through the construction of green financial system. China's goal of "carbon peaking and carbon neutrality" can be made possible driven with the transformation of green economy. Therefore, we should explore and establish a comprehensive evaluation system for the coordinated development of green finance and green economy. Five principles are used to select the evaluation indexes: First, the indexes that have been used more times in green economy with effective results. Second, it covers all sectors of green finance and green economy. Third, indexes that facilitate data collection, understanding and multicollinearity are selected. Fourth, we should abide by the legal provisions, policy opinions and guidance issued by the government and the industry. Fifth, we should learn from the mature international experience in green finance and green economy.

Since green finance and green economy are systematic and complex coupling and in order to master the cooperation, interaction and feedback among different influencing factors, we made exploration on the adoption of comprehensive index system and studied the coupling coordination between green finance and green economy system with combination of the coupling coordination degree model with ESG based on comprehensive adoption of "Carbon Cycle Necessary Support 
Mode". First, to improve the green financial system of financial service institutions, including green credit, loan pricing, economic capital allocation, resource utilization, financial support efficiency, environmental and social risk assessment, medium and long-term sustainable development indicators and other quantitative assessment indicators. Second, to improve the comprehensive risk management of green finance, and integrate ESG scoring system into asset quality management, product classification management and business process management of risk. Thirdly, to strengthen the evaluation and management of information disclosure, especially the investment environment and stock market value of listed companies. Fourth, to make evaluation on the strength of financial support by means of legislation, finance, taxation, finance, fund, credit, consultation and transaction.

The research shows that from the perspective of balanced development, the coordinated development of green finance and green economy in some countries, regions and operating entities is relatively high, which can realize the transition from barely balanced development to good balanced development, but there are still conditions of barely balanced development or even relatively lagging behind. From the perspective of support intensity, due to the differences of individual subjects and industries, the application effects of financial instruments also show different trends, which requires overall coordination. Compared with previous studies, the regional coupling coordination has a certain correlation, and the model's exploration of the relationship between green finance and green economy is innovative and enlightening.

\section{Suggestions on the coordination of green finance and green economy}

First, to improve the investment channels of green finance system and enhance the investment level. Second, to improve relevant policies to provide policy support for the development of green industry and promote the balanced development of the two elements. Thirdly, to establish the monitoring and feedback system, and improve the operating efficiency of the model through the positive feedback mechanism. Fourthly, to establish and improve the supervision system of the use of enterprise funds to strengthen the use of green special funds for improvement of the use efficiency. Fifthly, a feedback mechanism should be established to fully listen to the feedback on the effectiveness of the use of green finance policies. Elements of green finance and green economy should be added to the formulated industrial policies, and they should be updated and revised in a timely manner. To further strengthen policy support for carbon asset management (including CARBON capture and storage, carbon trading, and carbon recycling), battery recycling and reuse, waste sorting, and heating and cooling technology optimization;Sixth, green financial institutions should be guided to establish information service platforms, strengthen exchanges with green enterprises, better understand the financing needs of enterprises, and provide supporting financing consulting solutions. Seventh, to establish a green resource management system and incentive mechanism and build the whole process support model of carbon finance, establish a green market mechanism with a virtuous cycle so as to give play to the important role of carbon exchanges.

\section{Conclusion}

This study made explorations on how to better realize the coordinated development of green finance and green economy, and the paths of green finance and green economy as well as the dynamic coordination between them were further analyzed. By combining the coupling coordination degree model with ESG and the "carbon cycle necessary support mode", the development dynamic differences of individual industries are comprehensively evaluated, and appropriate methods are proposed to predict the long-term coordination degree and the coordination degree between green finance and green economy, to predict the degree of coordination between green finance and green economy, and to provide better policy suggestions for sustainable development. The international community is at a unique juncture. The recovery from COVID-19 offers an opportunity to tackle climate change and achieve net zero emissions. The world needs to enhance the coordination between green finance and green economy.

\section{References}

[1] Xie Ning. Improving green finance support for green development[N]. Study Times, 2021-07-14(003).

[2] Huang Xiangyao. Analysis on the mechanism of green finance development in Guangdong-Hong Kong-Macao Greater Bay Area[J]. China Journal of Commerce, 2021(13):1-3.

[3] Zheng Xiaolong, Zhao Xia. Green finance gives supports to the transformation of coal cities[J]. Modern Bankers, 2021(07):136-137.

[4] Li Yiping. Practical progress and breakthrough strategy of ecological service based economic development in China. Southwest Finance, 2021(07):27-38. 